\title{
A Facile Chemical Synthesis of PbTe Nanostructures at Room Temperature
}

\author{
Anil B. Gite 1,2®, Balasaheb M. Palve 1, Vishwasrao B. Gaikwad ${ }^{3}$, Gotan H. Jain ${ }^{2, *}$, \\ Habib M. Pathan ${ }^{1}{ }^{\mathbb{D}}$, Samir Haj Bloukh ${ }^{4}$ and Zehra Edis ${ }^{5, *}$ \\ 1 Advance Physics Laboratory, Department of Physics, Savitribai Phule Pune University, Pune 411 007, India; \\ gite.anil@gmail.com (A.B.G.); arjunpalve@gmail.com (B.M.P.); pathan@physics.unipune.ac.in (H.M.P.) \\ 2 SNJB'sArts, Commerce Science College, Chandwad, Nashik 423 101, India \\ 3 Department of Chemistry, KTHM College, Nashik 422 002, Maharashtra, India; \\ dr.gaikwadvb@rediffmail.com \\ 4 Department of Clinical Sciences, College of Pharmacy and Health Science, Ajman University, \\ Ajman PO Box 346, UAE; s.bloukh@ajman.ac.ae \\ 5 Department of Pharmaceutical Sciences, College of Pharmacy and Health Science, Ajman University, \\ Ajman PO Box 346, UAE \\ * Correspondence: gotanjain@rediffmail.com (G.H.J.); z.edis@ajman.ac.ae (Z.E.); Tel.: +971-56-694-7751 (Z.E.)
}

Received: 12 August 2020; Accepted: 23 September 2020; Published: 25 September 2020

\begin{abstract}
Thermoelectric (TE) materials are possible solutions of the current problems in the energy sector to overcome environmental pollution, increasing energy demand and the decline of natural resources. Thermoelectric materials are a promising alternative for the conversion of waste heat to electricity. Nanocrystalline PbTe powder was synthesized by a simple chemical method at room temperature and systematically investigated at various durations as samples A1-A5. Fourier Transform infrared spectroscopy (FTIR), x-ray diffraction (XRD), microstructural analysis by scanning electron microscopy (SEM), and energy dispersive spectroscopy (EDS) confirmed the composition of the samples. TE parameters as thermo-emf of samples A1-A5 and electrical conductivity were measured. The cyclic voltammetry gives a band gap of $0.25 \mathrm{eV}$, which is in agreement with the optical band gap of the material. The A4 sample has an average crystal size of $36 \mathrm{~nm}$ with preferred orientation in (200) verifying the cubic morphology. The obtained TE parameters are beneficial for the non-uniform TE materials which might be due to strong current boundary scattering and extremely low thermal conductivity of the samples.
\end{abstract}

Keywords: lead telluride; $\mathrm{PbTe}$; thermoelectric materials; chemical synthesis; nanostructures; cyclic voltammetry; electrical conductivity

\section{Introduction}

Thermoelectric materials can convert heat to electricity [1]. These materials are environment friendly and mostly used for the development of sustainable energy materials. It has been reported that the narrow band material like PbTe has shown superior thermoelectric (TE) properties and is currently used in different applications [2,3]. These materials have prospective uses in thermal power generation and thermal sensing equipments. Diverse theoretical calculations and experimental data point to the upgrading in TE properties, which can be achieved by reducing the dimensionality of TE materials to a certain lowest amount level [2,4-8]. The dimensionless parameter figure of merit, which determines the efficiency TE material is $\mathrm{ZT}=\left(\mathrm{S}^{2} \sigma \mathrm{T}\right) / \mathrm{K}$, where $\mathrm{S}$ is Seebeck coefficient, $\sigma$ is electrical conductivity, $\mathrm{T}$ is working temperature in Kelvin, and $\mathrm{K}$ is thermal conductivity of TE material [9]. A good TE material simultaneously demands large Seebeck coefficient (S), high electrical conductivity $(\sigma)$, and low 
thermal conductivity $(\mathrm{K})$. The thermal conductivity is due to the electron motion $(\mathrm{Ke})$ and lattice vibration produced within the material $\left(\mathrm{K}_{1}\right)$. This complex relationship between these TE parameters makes it difficult to enhance the ZT value of the TE materials. For that reason many efforts are aimed to improve the ZT value by balancing these interdependent TE parameters. Reducing the thermal conductivity via nanostructuring plays an important role for the improvement of the ZT of the material. For high ZT, high Seebeck coefficient is needed, but due to the complicated electronic band structures, superior electrical conductivity from high-symmetry cubic crystal structures, and low thermal conductivity caused by strong anharmonicity in TE materials, also due to the Pb local off-center, plays an important role in variations of TE parameters [10-12].

The quantum confinements will produce significant effects on charge and phonon transports in thermoelectric materials. Quantum confinement deals special way to manipulate carrier transports due to close connection between electronic band structure and dimensionality of the materials [13]. The Seebeck coefficient increased because of larger effective mass due to the band structure. $\mathrm{PbTe}$ material, indicating that reducing dimensionality of a given material is a potential possibility to enhance thermoelectric performance [14]. Studies of the film show a great interest in high performance and low dimensional TE materials since these materials perform better as compared to bulk materials [15]. PbTe is a great material for optoelectronic and in mid-infra-red ranges, also widely used in a large number of various TE devices [16]. The PbTe material also useful for the energy harvesting power generation [17,18]. Many thermoelectric materials are being explored for power generation applications, such as GeTe [19], PbTe [20,21], half-Heusler [22,23], and skutterudites [24,25]. Nanostructured materials that appear to be the most promising from a commercial point of view by virtue of their excellent thermoelectric performance and their high efficiency. The study thermoelectric nanomaterials are explored due to various approaches such as narrow bandgaps, heavy elements doping, point defects loading, and nanostructuring [26]. PbTe thin films were synthesized by various methods and have been utilized for research of PbTe material [27-34]. Wang et al. reported the simplistic chemical synthesis of $\mathrm{PbTe}$ on the glass substrate material at normal atmospheric conditions with temperatures around $300 \mathrm{~K}$ [35].

Chemical synthesis is one of the easiest methods for the synthesis of the material since it is a low-cost technique, which does not require any kind of sophisticated equipment, as well as specific kind of substrate materials. In the lead chalcogenide synthesis, $\mathrm{PbX}(\mathrm{PbX}, \mathrm{X}=\mathrm{Te}, \mathrm{S}$, Se) material films are synthesized by chemical technique [36-38]. Seleno-sulfate $\left(\mathrm{Na}_{2} \mathrm{SeSO}_{3}\right)$ is used widely as Se source by various groups for the synthesis of PbSe on glass substrate material [39-41]. Thiourea $\mathrm{SC}\left(\mathrm{NH}_{2}\right)_{2}$ or sodium thiosulfate $\left(\mathrm{Na}_{2} \mathrm{~S}_{2} \mathrm{O}_{3} \times \mathrm{H}_{2} \mathrm{O}\right)$ as sources of $\mathrm{S}$ are used for the synthesis of $\mathrm{PbS}$ on glass and silicon substrate materials [42-44].

Unfortunately, the corresponding Te source is difficult to obtain due to the scarce solubility of Te. Precursors like $\mathrm{TeO}_{2}, \mathrm{Na}_{2} \mathrm{TeO}_{3}$, and $\mathrm{Na}_{2} \mathrm{TeSO}_{3}$ are available, but unstable under natural conditions and therefore very rarely studies on chemical synthesis of $\mathrm{PbTe}$ are reported. In comparison to $\mathrm{S}$ or $\mathrm{Se}$, the dis-proportionating reactions and hydrolysis of Te in different $\mathrm{pH}$ solutions are much more difficult [45]. Further problems are the cost effectiveness, a maximum $\mathrm{ZT}_{\max }$, which is greater than 2.4 by the band convergence, and strain is produced in the lattice of $\mathrm{PbTe}$ [46]. Scarce and expensive material needed for the synthesis strain the opportunities to produce useful solutions for future energy problems. Recently, PbSe and PbS gained further attention due to their lower cost and higher operation temperature compared to Te [47,48].

However, the chemical synthesis of the PbTe material is easy and not frequently used due to the problem of hydrolysis of Te when compared to hydrothermal and solvothermal techniques, as well as solution phase synthesis and electro-deposition techniques [16,45,47,49-55].

In this study, we synthesized of $\mathrm{PbTe}$ powder by utilizing lead nitrate $\mathrm{Pb}\left(\mathrm{NO}_{3}\right)_{2}$ and tellurium oxide $\mathrm{TeO}_{2}$ as the precursors for $\mathrm{Pb}$ and $\mathrm{Te}$ from an alkaline aqueous solution bath. Lead telluride powder was prepared by a chemical method. High yield of PbTe for the various durations and formation mechanism of the PbTe powder and pellet was proposed and its TE properties of the film 
were measured for the better thermometric materials. We investigated in this work the synthesis of lead telluride powder by a chemical route technique. Silver-gray metallic powder of PbTe was successfully obtained at high yield. XRD reveals cubic nanocrystals of PbTe. The synthesized PbTe powder reveals homogeneous grains and agglomeration. The obtained values of the electrochemical band gap from the Cyclic Voltammetry are in agreement with the optical band gap. The formation of PbTe by chemical synthesis shall be a promising material that can be used as thermoelectric applications. Among the various synthesis techniques employed for the formation of PbTe nanostructures, chemical synthesis process has attracted much interest due to the advantage of high yield, low synthesis temperature, high purity, and high crystallinity.

\section{Materials and Methods}

\subsection{Materials}

Lead (II), telluride $\left(\mathrm{TeO}_{2}, 99.998 \%\right.$ trace metals basis), potassium hydroxide $(\mathrm{KOH})$, trisodium-citrate (TSC) and potassium borohydride $\left(\mathrm{KBH}_{4}\right)$ were purchased from Sigma Aldrich (St. Louis, MO, USA). Lead Nitrate $\left(\mathrm{Pb}\left(\mathrm{NO}_{3}\right)_{2}\right.$, 99\% pure) was obtained from Fisher Scientific (Pittsburgh, PA, USA). All the materials were pure and used as received. Additional purification was not done, when analytical grade precursors were utilized. Double distilled deionized water was used.

\subsection{Preparation of Samples}

In a typical run, $0.1 \mathrm{M} \mathrm{Pb}\left(\mathrm{NO}_{3}\right)_{2}, 0.1 \mathrm{M} \mathrm{TeO}_{2}, 2 \mathrm{M} \mathrm{KOH}, 0.2 \mathrm{M}$ tri-sodium citrate (TSC), and $0.8 \mathrm{M} \mathrm{KBH}_{4}$ were dissolved in sequence in $50 \mathrm{~mL}$ deionized water. Initially, a clean, colorless, transparent solution was formed. As time progressed, the color of the solution became dark gray and precipitation of dark gray material collected in the beaker for different durations as 72, 144, 216, 288 , and $360 \mathrm{~h}$. The solutions were then centrifuged for $10 \mathrm{~min}$ at $2000 \mathrm{rpm}$ in a centrifuge machine. After centrifugation, the samples were collected in dry crucibles. Finally, the sample was dried in a vacuum at a temperature of $500{ }^{\circ} \mathrm{C}$ for $30 \mathrm{~min}$. After the annealing, the collected grains were crushed to fine powder and the powder samples of different time durations were labeled as A1, A2, A3, A4, and A5. The samples were collected and analyzed for different characterization techniques and the TE properties of the samples were studied. Figure 1 represents the step-by-step procedure for the chemical bath deposition method used for the synthesis of PbTe.

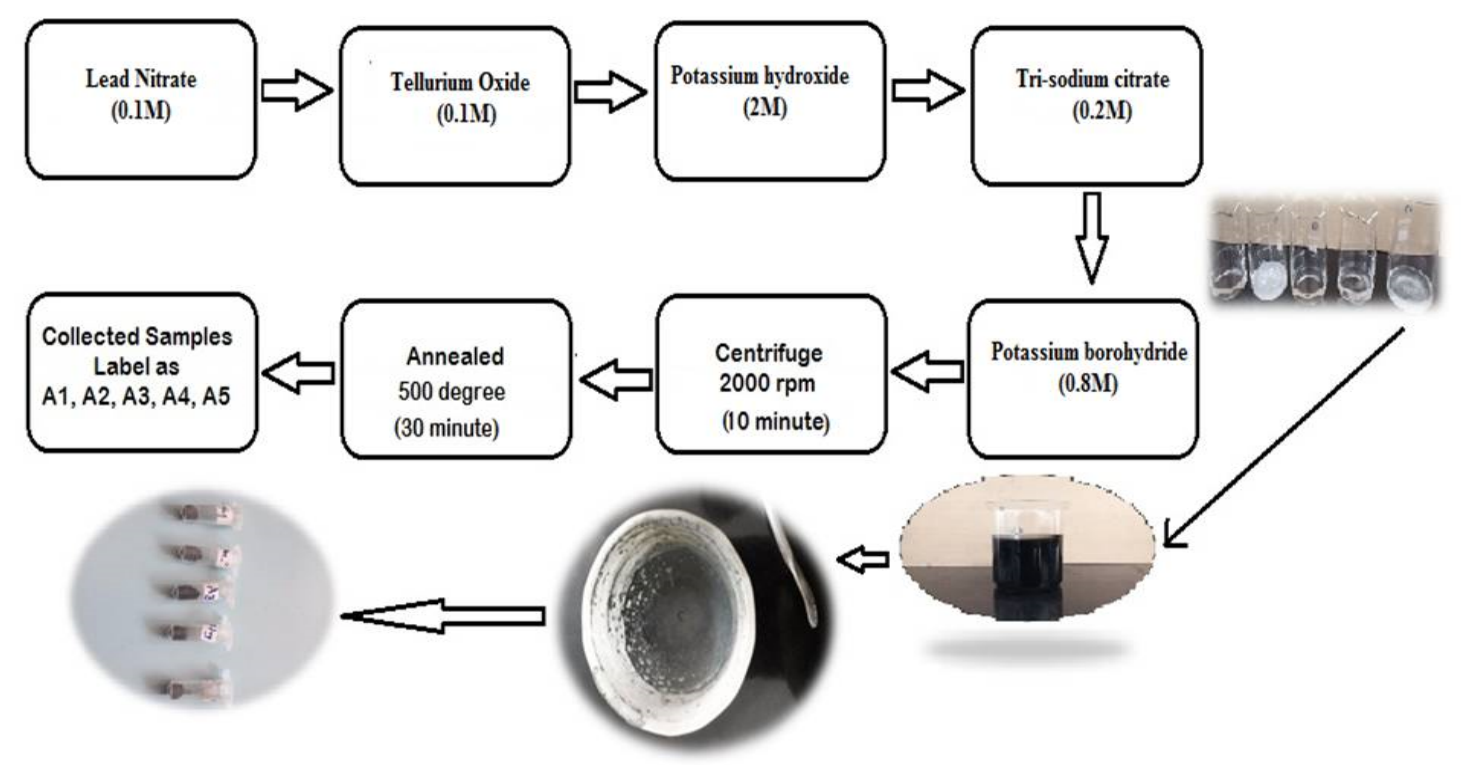

Figure 1. Step by step procedure for the synthesis of PbTe powder. 


\subsection{Characterization of Samples}

The samples A1-A5 were characterized by SEM/EDS, x-ray diffraction (XRD), FTIR, and cyclic voltammetry (CV). These methods confirmed the composition of our samples.

The PbTe structure was obtained by using the XRD-D8 Advance (Bruker, Karlsruhe, Germany) with $\mathrm{Cu}-\mathrm{K} \alpha$ line wavelength $1.54 \AA$. The morphological study of the synthesized material under higher magnification was done by SEM from JEOL (JSM-6400, Tokyo, Japan) with an accelerating voltage of $20 \mathrm{kV}$ of A1 and A5. The energy dispersive X-ray spectroscopy is used for the compositional analysis of the sample A1 and A5. For the FTIR analysis, JASCO FT/IR 6100 (Tokyo, Japan), with a range from 400 to $4000 \mathrm{~cm}^{-1}$ was used to find the presence of various modes in the material. Cyclic voltammetry (CV) measurements were carried out by K-Lyte 1.2 with the research applications of K-Lyte hardware (K-Lyte 1.2, from Knopy Techno Solutions, Kanpur, India). Seebeck coefficient measurements were done by a TEP unit TYPE-2, purchased from Borade Embedded Solutions, Kolhapur, India. Finally, for each sample, the pellets with dimensions $12 \mathrm{~mm} \times 3 \mathrm{~mm}$ were prepared by a hydraulic press machine to find the thermo-emf of the synthesized material.

\section{Results and Discussion}

\subsection{Characterization of Samples}

Qualitative analysis of the obtained yield was carried out by plotting the reaction time against the mass of the product (Figure 2).

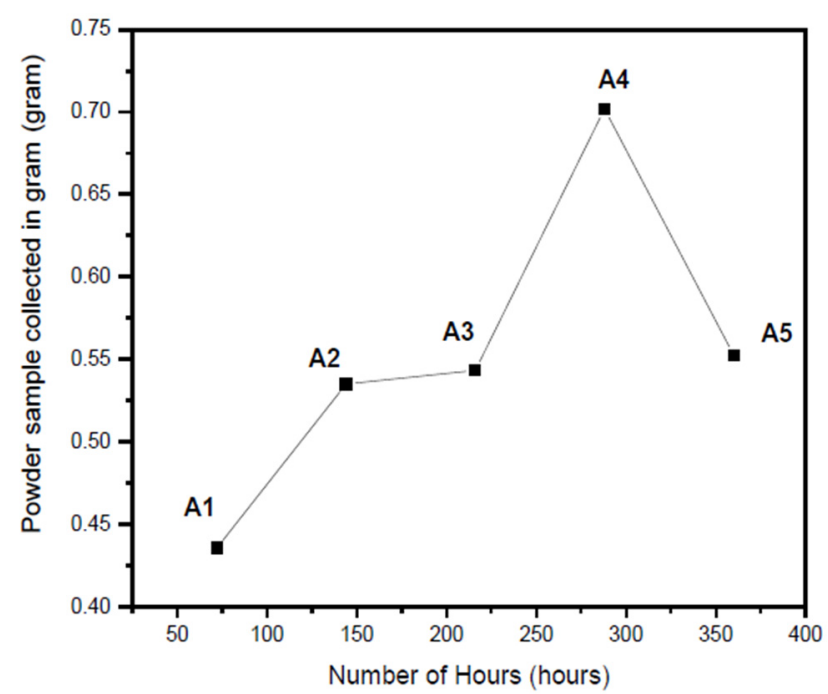

Figure 2. Yield of collected samples A1, A2, A3, A4, and A5 against the number of hours.

Lead nitrate and tellurium oxide were dissolved in excess alkali and formed $\mathrm{HPbO}^{2-}$ and $\mathrm{TeO}_{3}{ }^{2-}$ ions during the reaction time. These ions further precipitate at the bottom. As time progressed, the rate of reaction and formation of PbTe may have been enhanced. This can be evident from the maximum yield in the case of sample A4. However, a further increase in the reaction time may have led to the overgrowth and thus, resulted in the deteriorated yield in the case of sample A5.

\subsection{Cyclic Voltammetry (CV) Studies}

$\mathrm{CV}$ studies revealed the appropriate potential ranges of the ions in the given electrolyte solutions. The curves give the scanning of the electrolyte in the cathodic direction and the negative current produced is called the cathodic currents (Figure 3). 


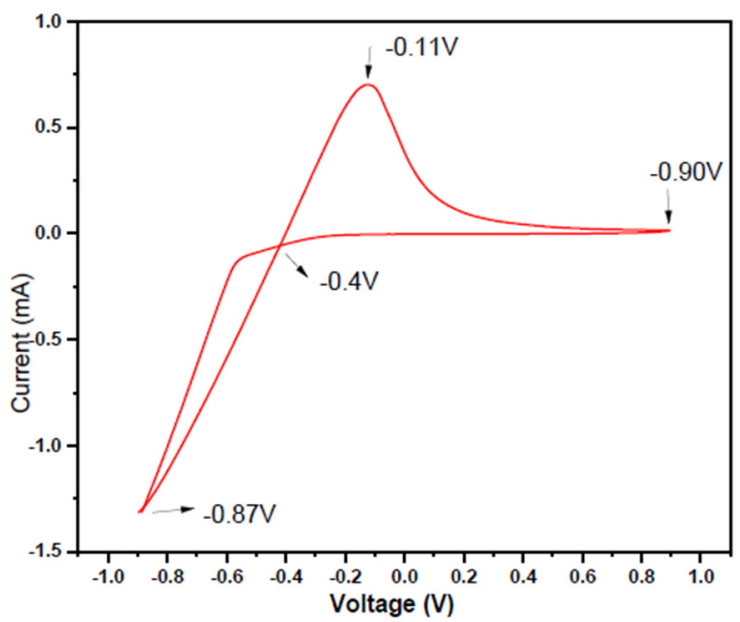

Figure 3. Cyclic Voltammogram of $0.1 \mathrm{M} \mathrm{Pb}\left(\mathrm{NO}_{3}\right)_{2}$ at a scan rate of $50 \mathrm{mV} / \mathrm{s}$ versus Pt tip working electrode and $\mathrm{Ag} / \mathrm{AgCl}$ as a reference electrode.

Figure 3 shows the deposition on the working electrode at a potential around $-0.87 \mathrm{~V}$ versus $\mathrm{Ag} / \mathrm{AgCl}$, in the cathodic scan. The potential becomes more negative to the anodic scan and a strong oxidation peak was observed at $-0.11 \mathrm{~V}$. The precursor solution of lead nitrate in water as:

$$
\mathrm{Pb}\left(\mathrm{NO}_{3}\right)_{2(\mathrm{~s})} \rightarrow \mathrm{Pb}_{\mathrm{aq}}{ }^{2+}+2 \mathrm{NO}_{3}{ }^{-} \mathrm{aq},
$$

with $\mathrm{Pb}_{\mathrm{aq}}{ }^{2+}$ ion has standard reduction potential $\left(\mathrm{E}^{0}\right)$ of $-0.125 \mathrm{~V}$ and $\mathrm{NO}_{3}{ }^{-}$aq ion in acidic conditions has $\mathrm{E}^{0}$ of $+0.956 \mathrm{~V}[43,56]$. The hysteresis was observed between the potential $-0.87 \mathrm{~V}$ to $+0.90 \mathrm{~V}$ indicating that reduction of $\mathrm{Pb}_{\mathrm{aq}}{ }^{2+}$ occurs more rapidly on the Pt tip working electrode. The potential was negatively shifted versus $\mathrm{Ag} / \mathrm{AgCl}$ and the reduction peak revealed the reduction of $\mathrm{Te}$ as shown in Figure 4.

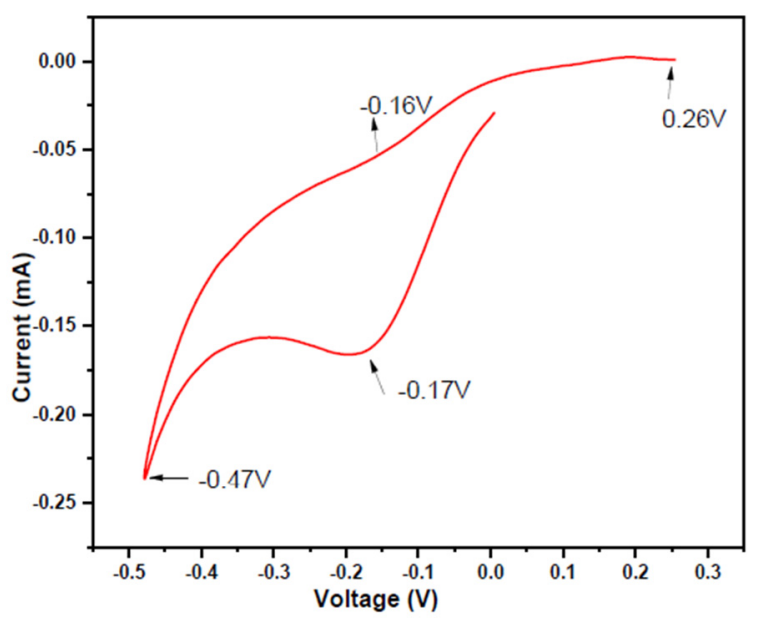

Figure 4. Cyclic Voltammogram of $0.1 \mathrm{mM} \mathrm{HTeO}^{2+}$ at scan rate of $50 \mathrm{mV} / \mathrm{s}$ versus Pt tip working electrode and $\mathrm{Ag} / \mathrm{AgCl}$ as a reference electrode.

The reduction of Te can be represented as:

$$
\mathrm{HTeO}_{2}^{-}+3 \mathrm{H}^{+}+4 \mathrm{e}^{-} \rightarrow \mathrm{Te}_{\mathrm{ads}}+2 \mathrm{H}_{2} \mathrm{O},
$$

where $\mathrm{Te}_{\text {ads }}$ indicates that Tellurium atoms are absorbed in an electrolyte solution. When the potential reaches $-0.17 \mathrm{~V}$, another reduction wave started, which is attributed to the production of $\mathrm{H}_{2} \mathrm{Te}$ [41]. 
Each of the reduction peaks were some sort of limited deposition. This behavior involves the slow reduction of $\mathrm{HTeO}^{2+}$. Cyclic Voltammetry shows the presence of semiconductor material that has a discrete and fixed energy level. The correlation between the optical and electrochemical band gap was reported the first time by Haram et al. [56]. Figure 5 gives the electrochemical band gap and it is calculated from the peak values.

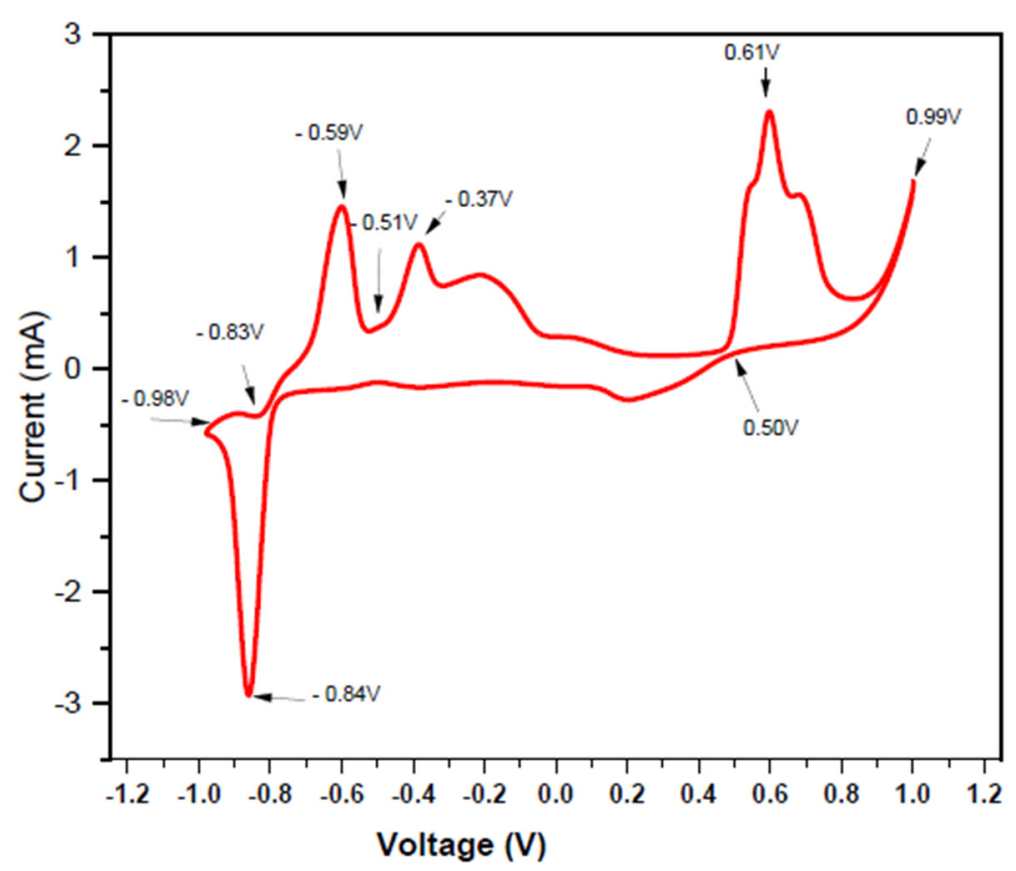

Figure 5. Cyclic Voltammetry of all the solutions when they are mixed together. Pt is used as working electrode, $\mathrm{Pt}$ mesh as a counter electrode and $\mathrm{Ag} / \mathrm{AgCl}$ as reference electrode for sweep rate of $50 \mathrm{mV} / \mathrm{s}$ from $-1.0 \mathrm{~V}$ to $+1.0 \mathrm{~V}$ at the current limit of $1 \mathrm{~mA}$ by using K-Lyte 1.2 Potentiostat. Cyclic Voltagram of $0.1 \mathrm{M} \mathrm{Pb}\left(\mathrm{NO}_{3}\right)_{2}, 0.1 \mathrm{M} \mathrm{TeO}_{2}, 2 \mathrm{M} \mathrm{KOH}, 0.2 \mathrm{M}$ trisodium citrate (TSC), and $0.8 \mathrm{M} \mathrm{KBH}_{4}$ were dissolved in sequence in $50 \mathrm{~mL}$ double distilled deionized water.

The obtained values are very well in agreement with an optical band gap:

$$
\Delta \mathrm{E}=\mathrm{E}_{\mathrm{ox}}-\mathrm{E}_{\text {red }}(\mathrm{eV}),
$$

Values from the cyclic voltammetry curve $\mathrm{E}_{\mathrm{ox}}=-0.59 \mathrm{~V}$ and $\mathrm{E}_{\text {red }}=-0.84 \mathrm{~V}$ give $\Delta \mathrm{E}=0.25 \mathrm{eV}$. The band gap of the PbTe is well matched with the observed band gap for the PbTe material.

\subsection{Structural Analysis}

The average crystallite size was calculated from XRD data which is based on Debye Scherrer's formula [57]:

$$
\mathrm{D}=0.9 \lambda / \beta \cos \theta,
$$

where $\mathrm{D}=$ average crystallite size, $\beta=$ broadening of the diffraction line measured at half maximum intensity (FWHM), $\lambda=$ wavelength of $X$-ray radiation, and $\theta=$ Bragg's angle. The calculated $d$-spacing and crystallite sizes corresponding to various crystal planes are presented in Table 1. Figure 6 shows the typical XRD pattern for samples A3, A4, and A5 annealed after $500{ }^{\circ} \mathrm{C}$. 
Table 1. X-ray diffraction (XRD) analysis of the PbTe powder for sample A4.

\begin{tabular}{|c|c|c|c|c|}
\hline (h k l) & 2 (Degree) & d Spacing $(\AA ̊)$ & FWHM $\left(\times 10^{-3}\right)$ & Crystallite Size (nm) \\
\hline$\left(\begin{array}{lll}1 & 1 & 0\end{array}\right)$ & 24.15 & 3.72 & 2.004 & 42.37 \\
\hline$\left(\begin{array}{lll}2 & 0 & 0\end{array}\right)$ & 27.94 & 3.22 & 2.004 & 42.70 \\
\hline$\left(\begin{array}{lll}2 & 2 & 0\end{array}\right)$ & 39.91 & 2.28 & 4.004 & 22.04 \\
\hline$\left(\begin{array}{llll}3 & 1 & 1\end{array}\right)$ & 49.10 & 1.86 & 2.004 & 45.55 \\
\hline (2 2 2 1$)$ & 57.30 & 1.61 & 4.008 & 23.61 \\
\hline$\left(\begin{array}{lll}4 & 2 & 0\end{array}\right)$ & 64.60 & 1.44 & 2.004 & 49.02 \\
\hline$\left(\begin{array}{lll}4 & 2 & 2\end{array}\right)$ & 72.04 & 1.31 & 4.008 & 25.62 \\
\hline
\end{tabular}

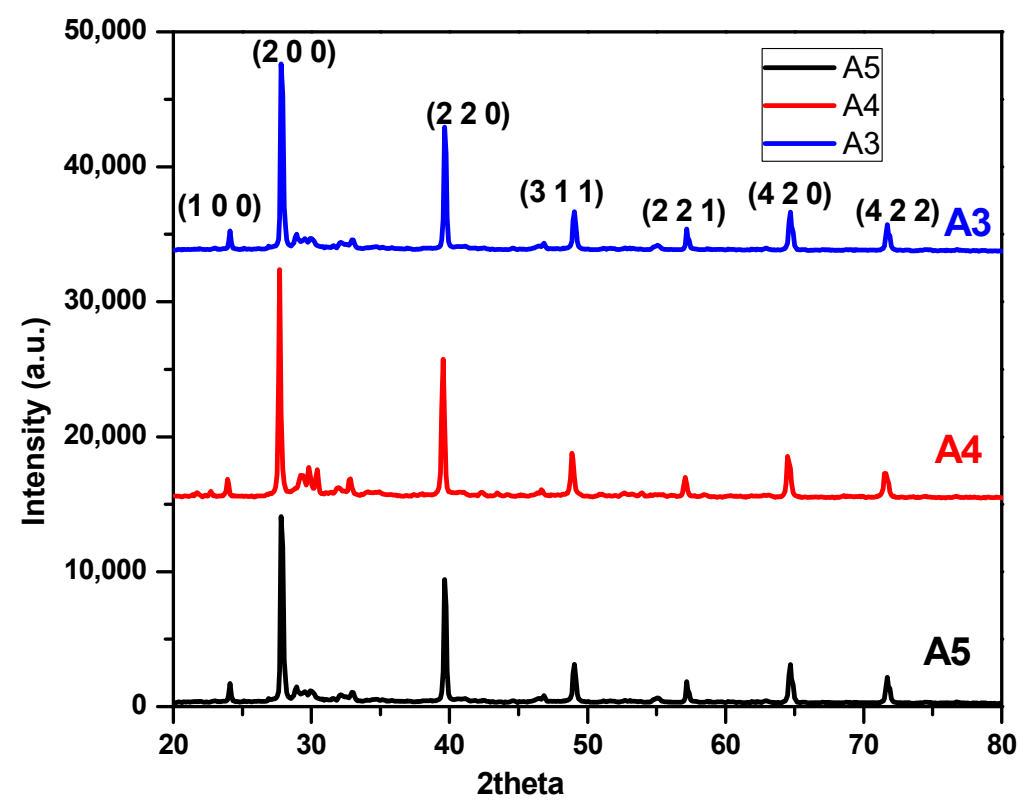

Figure 6. X-ray diffraction (XRD) analysis of samples A3, A4, and A5.

All the diffraction signatures along the ( $\mathrm{h} \mathrm{k}$ ) plane can be attributed to the PbTe crystals. The powder samples A3, A4, and A5 give the well-matched peaks of their XRD patterns. The peak corresponding to $(200)$ and (2 20$)$ are the most prominent peaks as per the JCPDS file number 077-02460. Further strong intense peaks and other weak peaks are also indexed in the XRD pattern matching with the corresponding JCPDS file. The observed peaks were well matched with the XRD of undoped PbTe synthesized by solvothermal/hydrothermal process [58]. With the XRD of maximum yield sample A4 shows the formation of $\mathrm{PbTe}$ and reveals an average crystallite size of $36 \mathrm{~nm}$ for the A4 sample (Table 1).

The intensity and FWHM of XRD along (2 00 ) matches with the standard XRD pattern and thus, can be attributed to the prominent peak indicating the formation of the PbTe crystal in all the samples. This suggests that few crystals have followed the preferred orientation along $(200)$. This may be evident from the independent small cubic morphologies shown in scanning electron micrographs. However, the XRD reveals additional weak orientations along (4 20 ) and (4 22 2) planes, which may have led to the agglomeration of the cubes in the bunch like morphologies. The dislocation density is defined as the length of dislocations lines per unit volume of crystal and calculated from the formula [59] as $\delta=1 / \mathrm{D}^{2}$ where $\mathrm{D}$ is the crystal size and the micro-strain $\varepsilon$ is given by $\varepsilon=\beta \cos \theta / 4$. For the synthesized material the calculated values for dislocation density are $7.74 \times 10^{14}$ and micro-strain produced in the synthesized material is $2.41 \times 10^{-3}$. 


\subsection{Scanning Electron Microscope (SEM) and Elemental Studies}

Microstructural analysis by SEM/EDS was utilized for the morphological analysis of the samples, their sizes, and their composition. Figure 7 gives typical SEM images of sample A1 and A5 obtained at different magnifications.
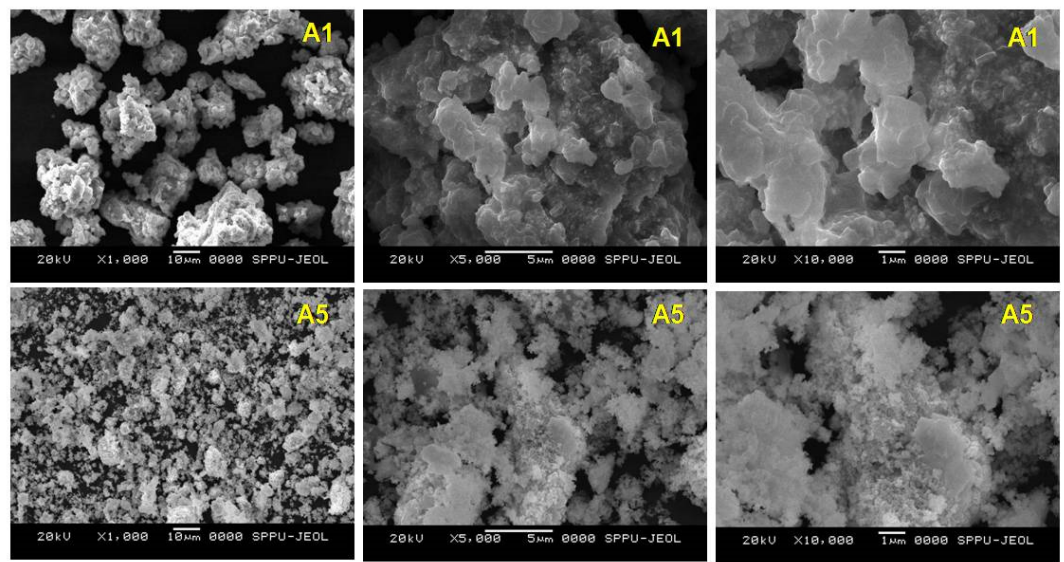

Figure 7. Scanning Electron Microscope (SEM) images of the sample at various magnifications of sample preparation.

SEM reveals the formation of bunches of independent cubes. This may be attributed to the enhanced rate of reaction as a function of reaction time. Agglomerations of PbTe particles take place as the time progress as seen in the SEM images of sample A5. Elemental spectra of both the sample A1 and $\mathrm{A} 5$ reveal the strong peaks of $\mathrm{Pb}$ and $\mathrm{Te}$ as shown in Figure 8.
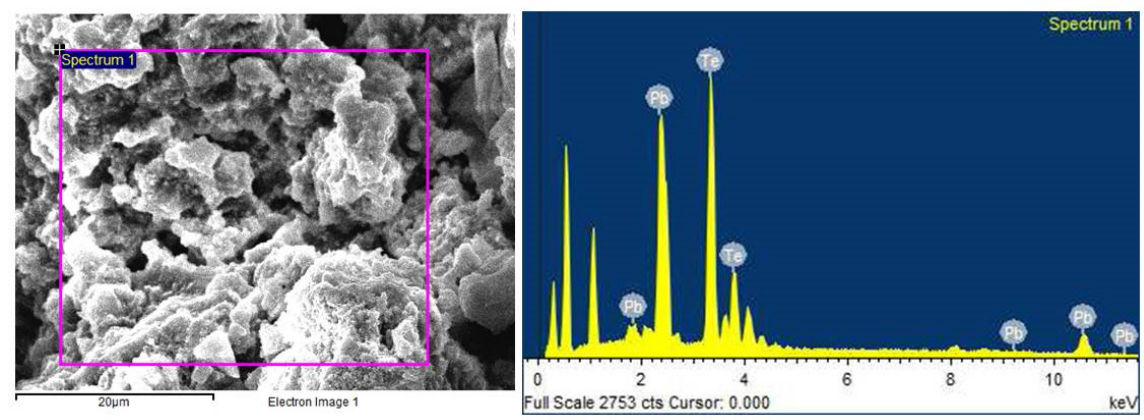

(a)
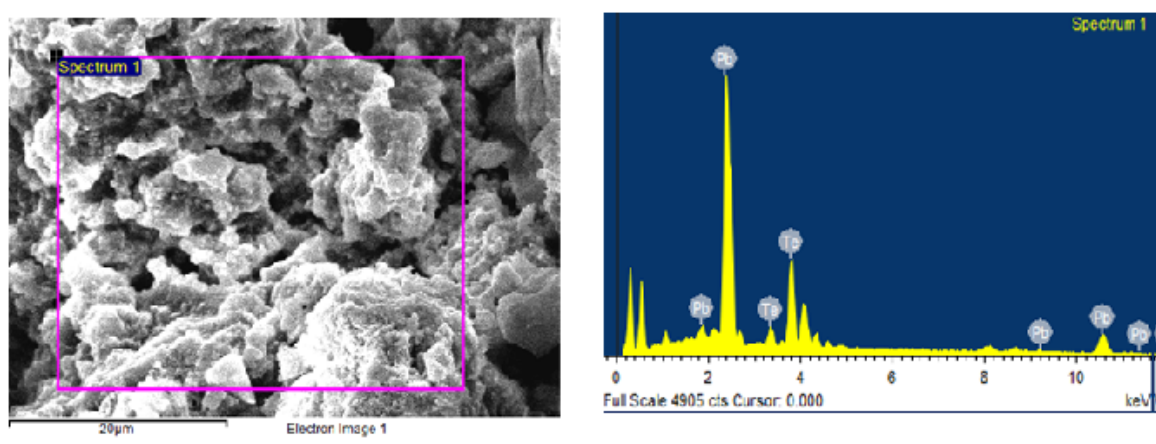

(b)

Figure 8. Energy dispersive spectroscopy (EDS) and corresponding scanning electron microscopy (SEM) of two samples. From up to down: (a) A1; (b) A5. 
Besides, the observed additional peaks may be attributed to the physical adsorption of oxygen in the sample in air. Table 2 shows the composition of the A1 and A5 samples.

Table 2. Elemental content of samples A1 and A5.

\begin{tabular}{ccccc}
\hline Sample & Element & Te (L) & Pb (M) & Total \\
\hline \multirow{2}{*}{ A1 } & Weight \% & 7.82 & 15.25 & 23.06 \\
& Atomic \% & 45.43 & 54.57 & 100 \\
A5 & Weight \% & 30.76 & 16.69 & 46.47 \\
& Atomic \% & 53.15 & 46.85 & 100 \\
\hline
\end{tabular}

At shorter durations in the sample $\mathrm{A} 1$, the amount of Te was less than the amount of $\mathrm{Pb}$ with $45.43 \%$ and $54.57 \%$, respectively. With increasing duration in sample A5, this changed to the opposite with increasing atomic percent of $\mathrm{Te}(53.15 \%)$ compared to $\mathrm{Pb}(46.85 \%)$, according to Table 2. The stoichiometric composition with atomic percentages should be in samples A1 and A5.

\subsection{Fourier-Transform Infrared Spectroscopy (FTIR)}

The optical characterization of prepared samples was performed with FTIR transmission (\%T) measurements. FTIR analysis of the synthesized PbTe samples confirmed their composition and indicates the presence of various modes of PbTe in samples A1 and A5 (Figure 9). The organic or inorganic nature of the samples can be identified by using this technique for sample A1. The bands at $1409 \mathrm{~cm}^{-1}, 1562 \mathrm{~cm}^{-1}, 2885 \mathrm{~cm}^{-1}$, and $2813 \mathrm{~cm}^{-1}$ belong to the $-\mathrm{CH}_{2}$ outplane swinging, asymmetric (COO-) and symmetric $-\mathrm{CH}_{2}$ stretches in sample A1 (Figure 9). The bands at $2961 \mathrm{~cm}^{-1}$ and $2813 \mathrm{~cm}^{-1}$ are due to the $-\mathrm{C}-\mathrm{H}$ stretching and anti-stretching vibrations of the $-\mathrm{CH}_{2}$ group, respectively, for the sample A1. This confirms the presence of stretching and bending modes of PbTe in the synthesized films. The bands around $1107 \mathrm{~cm}^{-1}$ to $1409 \mathrm{~cm}^{-1}$ might originate from $-\mathrm{C}-\mathrm{N}$ stretching, $-\mathrm{CH}_{2}$ scissoring mode of vibration, and $-\mathrm{OH}$ bending mode. The bands between $781-907 \mathrm{~cm}^{-1}$ reveal vibrations of ternary amines with $-\mathrm{CH}_{2}$ rocking modes [54,60,61].

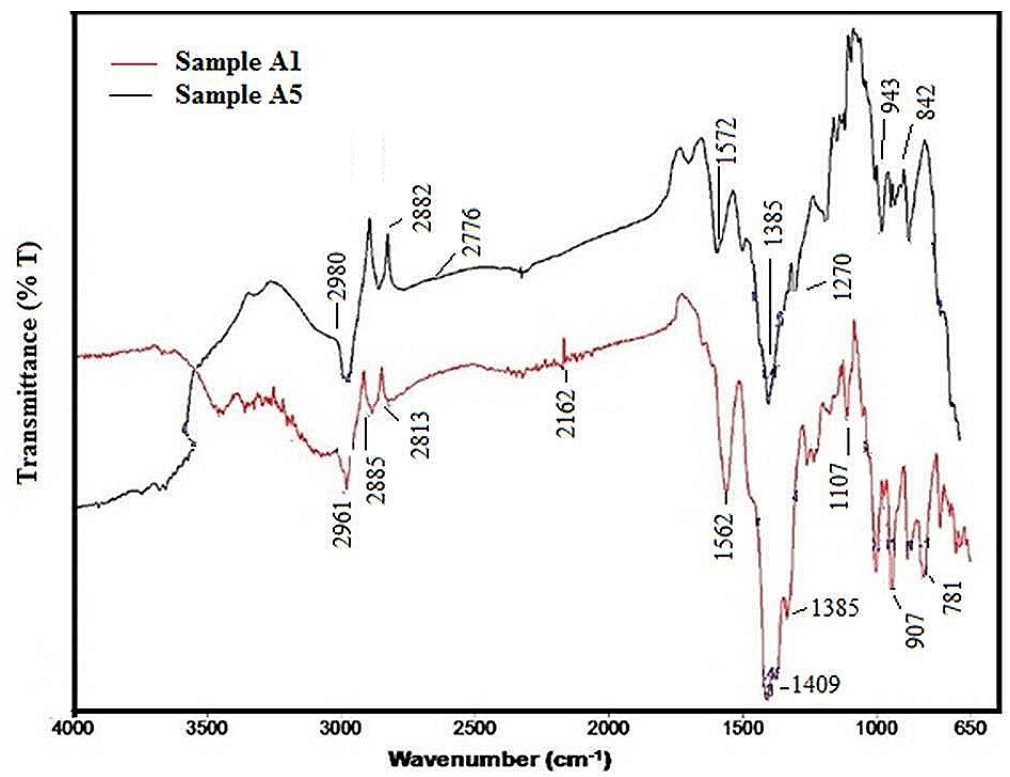

Figure 9. Fourier-transform-infrared (FTIR) spectrometric analysis of samples A1 and A5.

FTIR spectra of the sample A5 show that the strong absorption bands at $1572 \mathrm{~cm}^{-1}, 1270 \mathrm{~cm}^{-1}$, and $1385 \mathrm{~cm}^{-1}$ are attributed to stretching vibrations of the carboxylate groups in the film. Various bands at $2980 \mathrm{~cm}^{-1}, 2882 \mathrm{~cm}^{-1}$, and $2776 \mathrm{~cm}^{-1}$ are due to $-\mathrm{CH}$ stretching vibrations present in the structure 
of the $\mathrm{PbTe}$ [60]. The FTIR spectrum of the PbTe sample prepared by chemical precipitation at room temperature for $288 \mathrm{~h}$ of sample A5, indicates the presence of the bond of $\mathrm{Pb}-\mathrm{Te}$. The FTIR spectrum does not show strong bands associated with $\mathrm{Pb}-\mathrm{Se}$ stretching, and bending vibrations, nor $-\mathrm{CH}_{2}$ Scissoring mode vibration, $-\mathrm{C}-\mathrm{N}$ stretching, and $-\mathrm{OH}$ bending mode [61]. The vibrations of ternary amines are available at 842 to $943 \mathrm{~cm}^{-1}$ and the $-\mathrm{CH}_{2}$ rocking modes are observed between the $1270-1572 \mathrm{~cm}^{-1}$. FTIR transmittance bands are summarized in Table 3. The FTIR spectra of sample well matched with those reported earlier [54,60-63].

Table 3. Fourier-transform-infrared (FTIR) spectrometric analysis of PbTe samples of sample A5.

\begin{tabular}{cccccc}
\hline Peak No. & $\begin{array}{c}\mathbf{X} \\
\left(\mathbf{c m}^{-\mathbf{1}}\right)\end{array}$ & $\begin{array}{c}\mathbf{Y} \\
(\mathbf{\%})\end{array}$ & Peak No. & $\begin{array}{c}\mathbf{X} \\
\left(\mathbf{c m}^{-\mathbf{1}}\right)\end{array}$ & $\begin{array}{c}\mathbf{Y} \\
(\mathbf{\%})\end{array}$ \\
\hline 1 & 2980.04 & 52.10 & 6 & 1270.56 & 58.49 \\
2 & 2882.32 & 56.13 & 7 & 1152.32 & 62.51 \\
3 & 2776.28 & 56.17 & 8 & 943.53 & 62.38 \\
4 & 1572.67 & 58.11 & 9 & 908.60 & 63.56 \\
5 & 1385.47 & 50.94 & 10 & 842.71 & 61.07 \\
\hline
\end{tabular}

\subsection{Thermo-Electromotive Force Measurements}

The thermo-electrical properties were studied in previous investigations [63]. The Seebeck coefficient of PbTe was measured by mounting the sample on two metal blocks, which enabled the generation of a temperature gradient, as reported in the previous studies [64]. For all measurements, one side contact was kept at room temperature (approximately $25^{\circ} \mathrm{C}$ ) with the help of circulating water at one side contact, while the other one was heated allowing the generation of a temperature gradient. A copper contact has been around the samples contact, in order to conduct the thermoelectrically driven voltage measurements. Silver paste was present between the copper contact and the sample surface. The copper contact from the two ends were fed to a multimeter-voltmeter and the generated thermo-voltage $(\mathrm{V})$ was measured. The temperature gradient $\Delta \mathrm{T}$ was measured. Then the Seebeck coefficient (S) was derived from the ratio of $\Delta \mathrm{V} / \Delta \mathrm{T}$. In the synthesis of the powder, pellets of the dimensions 12 (diameter) $\times 3$ (height) $\mathrm{mm}$ were used in the measurements of the Seebeck coefficient $(S)$ and electrical conductivity $(\sigma)$ (Figure 10). Seebeck coefficient, as determined by laboratory made measurements system purchased from the Borade Embaded Solutions, provided the contacts mentioned above by using pellets formed from an exact size of the samples (A1-A5) respectively. The circular pellets of the dimensions were prepared by the hydraulic press machine, by application of high stress. Then, the thermo-emf of the samples was measured.

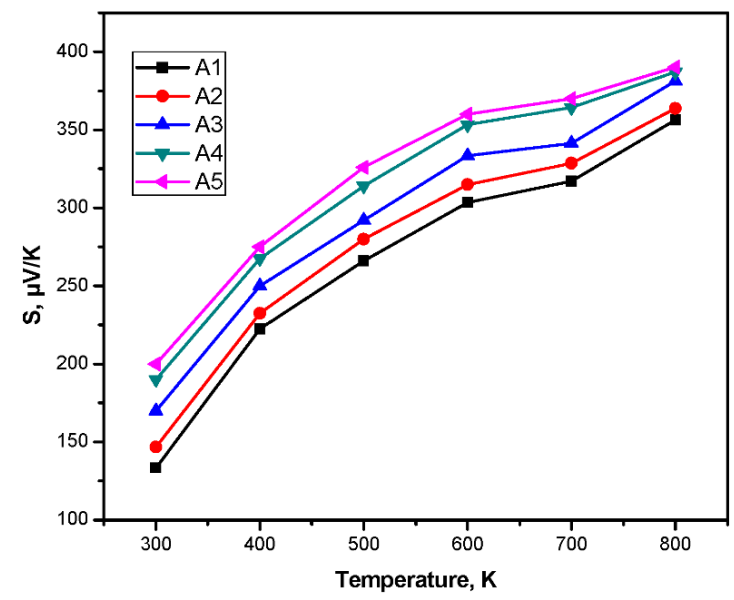

Figure 10. Plot of thermo-electromotive force versus temperature difference from two points on the pellets. 
Seebeck coefficient $(S)$ for the samples A1 to A5 shows the p-type behavior with the variation of $\mathrm{S}$ from $50 \mu \mathrm{VK}^{-1}$ to $380 \mu \mathrm{VK}^{-1}$. The values of $\sigma$ and $S$ for the high yield sample were $23 \mathrm{Sm}^{-1}$ and $314 \mu \mathrm{VK}^{-1}$, respectively, and varied with temperature. Figure 10 shows the non-linear behavior of $\mathrm{S}$ with temperature T. These values are much in comparison to the gas evaporation method reported [65]. From the samples A1 to A5, there is increase in the thermo-emf as seen, since sample A5 shows the agglomeration at longer time durations in the synthesized sample. The sample A4 resulted in these values of $\sigma$ and $S$ due to strong grain boundary scattering of carriers, which may lead to extremely low thermal conductivity $\mathrm{K}$ for the synthesized material. This is more beneficial for the non-dimensional TE materials. With increase in thermo-emf, the electrical conductivity should also increase. The figure of merit, $\mathrm{ZT}\left(\mathrm{ZT}=\mathrm{S}^{2} \sigma \mathrm{T} / \mathrm{k}\right)$, determines the TE conversion efficiency of the TE material. The TE properties of the material could be increased by doping the other elements such as Se, Sn, Sb, and Ag. The decreased value of $S$ is also observed when the PbTe is synthesized by the other methods and increase in the power factor of the material $[66,67]$. Figure 11 shows the schematic diagram of the Seebeck coefficient measurement.

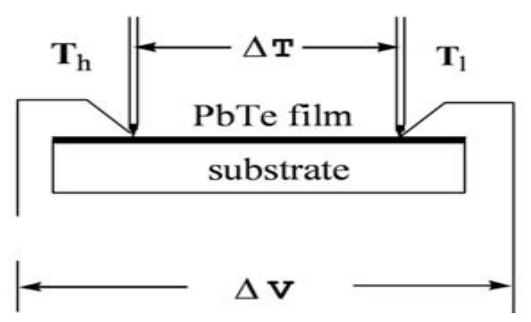

Figure 11. Schematic diagram of the Seebeck coefficient measurement.

As a result, the sample A4 is the best sample considering the yield produced and the measured values of the $\sigma$ and $S$ of the high yield sample. The synthesized PbTe pellet of high yield sample shows p-type conduction with electrical conductivity and Seebeck coefficient (S) as $23 \mathrm{Sm}^{-1}$ and $314 \mu \mathrm{VK}^{-1}$, respectively. This verifies that the higher quality of lead telluride material is formed at high yield. The plots of thermo-electromotive force versus temperature difference for all the samples from two points on the pellet film material indicate that the material shows the typical semiconducting of p-type behavior, and at higher temperature, the Seebeck coefficient of the samples almost remains the constant and decreasing nature. The sudden increase and decrease in the value of the thermo-emf indicates that the structural phase transition in the material will be observed during these temperature ranges of $500 \mathrm{~K}$ to $700 \mathrm{~K}$ (Figure 10).

\section{Conclusions}

Nanocrystalline PbTe powder was synthesized by a simple chemical method at room temperature and systematically investigated at various durations. This is a novel technique to synthesize the PbTe material which shows noble physico-chemical properties. Depending on the duration, samples were labelled as A1, A2, A3, A4, and A5. There is a direct relation between duration and yield of the sample until $288 \mathrm{~h}$. After $288 \mathrm{~h}$, the yield of sample A4 declined due to agglomeration. The cyclic voltammetry gives a band gap of $0.25 \mathrm{eV}$, which is very well in agreement with the optical band gap of the material. As compared to the other samples, the most prominent peaks of A4 sample in XRD pattern give the average crystal and size of $36 \mathrm{~nm}$ having preferred orientation in (2 00$)$, verifying the cubic morphology. The dislocation density of the A4 sample is calculated as having a value of $7.74 \times 10^{14}$ and the micro strain produced in the sample is $2.41 \times 10^{-3}$. The lower yield of A1 and high yield of A5 sample surface is a result of the formation of independent cubes of PbTe having different magnifications in SEM studies. In the sample A5, as time progressed, the agglomeration of particles took place with the decrease in the yield. The various stretches produced in the lower yield A1 and maximum time duration A5 samples are observed in FTIR stretches, giving the presence of PbTe with various modes of vibrations in transmission spectra. The plots of thermo-electromotive force versus 
temperature difference for all the samples from two points on the films indicate that the film is a p-type conductor. The thermo-emf of all samples were measured to show the constant thermo-emf for all the samples, but higher yield sample A4 gives the well agreement values of electrical conductivity and thermo-emf as compared to the other samples. These values are beneficial for the non-uniform TE materials which might be due to strong current boundary scattering and extremely low thermal conductivity of the sample.

Author Contributions: Conceptualization, H.M.P., G.H.J., and A.B.G.; methodology, A.B.G. and H.M.P.; software, B.M.P. and H.M.P.; validation, Z.E., S.H.B., and H.M.P.; formal analysis, H.M.P., G.H.J., and V.B.G.; investigation, H.M.P., G.H.J., A.B.G., and V.B.G.; resources, Z.E. and S.H.B.; data curation, H.M.P., S.H.B., and Z.E.; writing-original draft preparation, Z.E. and A.B.G.; writing-review and editing, Z.E., S.H.B., A.B.G., and H.M.P.; visualization, H.M.P., Z.E., and S.H.B.; supervision, H.M.P., S.H.B., and Z.E.; project administration, H.M.P., S.H.B., and Z.E. All authors within this team, conducted literature research and data interpretation additionally to the above mentioned contributions. All authors have read and agreed to the published version of the manuscript.

Funding: This research received no external funding.

Acknowledgments: Authors are thankful to Savitribai Phule Pune University Department of Physics for various characterization facilities; A.B.G. is grateful to Principal SNJB's ACS College for providing the necessary facility to carry out the research work; Principal Sandip Institute of Pharmaceutical Sciences (SIPS) for the use of the FTIR facility. Z.E. and S.H.B. are grateful to the Deanship of Graduate Studies and Research, AU, Ajman, United Arab Emirates.

Conflicts of Interest: The authors declare no conflict of interest.

\section{References}

1. Feng, Y.; Witkoske, E.; Bell, E.S.; Wang, Y.; Tzempelikos, A.; Ferguson, I.T.; Lu, N. Advanced Metal Oxides and Nitrides Thermoelectric Materials for Energy Harvesting. ES Mater. Manuf. 2018, 1, 13-20. [CrossRef]

2. Freer, R.; Powell, A.V. Realising the potential of thermoelectric technology: A Roadmap. J. Mater. Chem. C 2020, 8, 441-463. [CrossRef]

3. Soleimani, Z.; Zoras, S.; Ceranic, B.; Shahzad, S.; Cui, Y. A review on recent developments of thermoelectric materials for room-temperature applications. Sustain. Energy Technol. Assess. 2020, 37, 100604. [CrossRef]

4. Beretta, D.; Neophytou, N.; Hodges, J.M.; Kanatzidis, M.G.; Narducci, D.; Martin-Gonzalez, D.; Beekman, M.; Balke, B.; Cerretti, G.; Tremel, W.; et al. Thermoelectrics: From history, a window to the future. Mater. Sci. Eng. R Rep. 2019, 138, 100501. [CrossRef]

5. Harman, T.C.; Taylor, P.J.; Walsh, M.P.; LaForge, B.E. Quantum Dot Superlattice Thermoelectric Materials and Devices. Science 2002, 297, 2229-2232. [CrossRef]

6. Wolf, M.; Hinterding, R.; Feldhoff, A. High Power Factor vs. High zT-A Review of Thermoelectric Materials for High-Temperature Application. Entropy 2019, 21, 1058. [CrossRef]

7. Hasdeo, E.H.; Krisna, L.P.A.; Hanna, M.Y.; Gunara, B.E.; Hung, N.T.; Nugraha, A.R.T. Optimal band gap for improved thermoelectric performance of two-dimensional Dirac materials. J. Appl. Phys. 2019, 126, 035109. [CrossRef]

8. Hicks, L.D.; Harman, T.C.; Sun, X.; Dresselhaus, M.S. Experimental study of the effect of quantum-well structures on the thermoelectric figure of merit. Phys. Rev. B 1996, 53, R10493. [CrossRef]

9. Disalvo, F.J. Thermoelectric cooling and power generation. Science 1999, 285, 703-706. [CrossRef]

10. Božin, E.S.; Malliakas, C.D.; Souvatzis, P.; Proffen, T.; Spaldin, N.A.; Kanatzidis, M.G.; Billinge, S.J.L. Entropically stabilized local dipole formation in lead chalcogenides. Science 2010, 330, 1660-1663. [CrossRef]

11. Delaire, O.; Ma, J.; Marty, K.; May, A.F.; McGuire, M.A.; Du, M.H.; Singh, D.J.; Podlesnyak, A.; Ehlers, G.; Lumsden, M.D.; et al. Giant anharmonic phonon scattering in PbTe. Nat. Mater. 2011, 10, 614-619. [CrossRef]

12. Chang, C.; Zhao, L.D. Anharmoncity and low thermal conductivity in thermoelectrics. Mater. Today Phys. 2018, 4, 50-57. [CrossRef]

13. Zhang, X.; Pei, Y. Manipulation of charge transport in thermoelectrics. NPJ Quantum Mater. $2017,2$. [CrossRef]

14. Xiao, Y.; Zhao, L.D. Charge and phonon transport in PbTe-based thermoelectric materials. NPJ Quantum Mater. 2018, 3. [CrossRef] 
15. Harman, T.C.; Taylor, P.J.; Spears, D.L.; Walsh, M.P. Thermoelectric quantum-dot superlattices with high ZT. J. Electron. Mater. 2000, 29, L1-L2. [CrossRef]

16. Tan, G.; Ohta, M.; Kanatzidis, M.G. Thermoelectric power generation: From new materials to devices. Philos. Trans. R. Soc. A Math. Phys. Eng. Sci. 2019, 377, 20180450. [CrossRef]

17. Petsagkourakis, I.; Tybrandt, K.; Crispin, X.; Ohkubo, I.; Satoh, N.; Mori, T. Thermoelectric materials and applications for energy harvesting power generation. Sci. Technol. Adv. Mater. 2018. [CrossRef] [PubMed]

18. Sahay, S.S.; Guruswamy, S. Epitaxial growth of PbTe film on Si substrate. J. Mater. Sci. Lett. 1998. [CrossRef]

19. Wu, D.; Zhao, L.D.; Hao, S.; Jiang, Q.; Zheng, F.; Doak, J.W.; Wu, H.; Chi, H.; Gelbstein, Y.; Uher, C.; et al. Origin of the high performance in GeTe-based thermoelectric materials upon $\mathrm{Bi}_{2} \mathrm{Te}_{3}$ doping. J. Am. Chem. Soc. 2014, 32, 1693-1701. [CrossRef]

20. Hsu, K.F.; Loo, S.; Guo, F.; Chen, W.; Dyck, J.S.; Uher, C.; Hogan, T.; Polychroniadis, E.K.; Kanatzidis, M.G. Cubic $\mathrm{AgPb}_{\mathrm{m}} \mathrm{SbTe}_{2+\mathrm{m}}$ : Bulk Thermoelectric Materials with High Figure of Merit. Science 2004, 303, 818-821. [CrossRef]

21. Lalonde, A.D.; Pei, Y.; Wang, H.; Jeffrey Snyder, G. Lead telluride alloy thermoelectrics. Mater. Today 2011, 303, 818-821. [CrossRef]

22. Rull-Bravo, M.; Moure, A.; Fernández, J.F.; Martín-González, M. Skutterudites as thermoelectricmaterials: Revisited. RSC Adv. 2015, 5, 41653-41667. [CrossRef]

23. Shi, X.; Yang, J.; Salvador, J.R.; Chi, M.; Cho, J.Y.; Wang, H.; Bai, S.; Yang, J.; Zhang, W.; Chen, L. Multiple-filled skutterudites: High thermoelectric figure of merit through separately optimizing electrical and thermal transports. J. Am. Chem. Soc. 2011, 133, 7837-7846. [CrossRef]

24. Xing, Y.; Liu, R.; Liao, J.; Zhang, Q.; Xia, X.; Wang, C.; Huang, H.; Chu, J.; Gu, M.; Zhu, T.; et al. High-efficiency half-Heusler thermoelectric modules enabled by self-propagating synthesis and topologic structure optimization. Energy Environ. Sci. 2019, 12, 3390-3399. [CrossRef]

25. Xin, J.; Tang, Y.; Liu, Y.; Zhao, X.; Pan, H.; Zhu, T. Valleytronics in thermoelectric materials. NPJ Quantum Mater. 2018, 3. [CrossRef]

26. Chen, Z.G.; Hana, G.; Yanga, L.; Cheng, L.; Zou, J. Nanostructured thermoelectric materials: Current research and future challenge. Prog. Nat. Sci. Mater. Int. 2012, 22, 535-549. [CrossRef]

27. Nashed, G. Annealing temperature effect on properties of chemically deposited PbTe films and bulk. Mat. Sci. Indian J. 2015, 13, 325-335. [CrossRef]

28. Hicks, L.D.; Dresselhaus, M.S. Effect of quantum-well structures on the thermoelectric figure of merit. Phys. Rev. B 1993, 47, 12727-12731. [CrossRef]

29. Poudeu, P.F.R.; D'Angelo, J.; Downey, A.D.; Short, J.L.; Hogan, T.P.; Kanatzidis, M.G. High Thermoelectric Figure of Merit and Nanostructuring in Bulk p-type $\mathrm{Na}_{1-\mathrm{x}} \mathrm{Pb}_{\mathrm{m}} \mathrm{Sb}_{\mathrm{y}} \mathrm{Te}_{\mathrm{m}+2}$. Angew. Chem. Int. Ed. 2006, 45, 3835-3839. [CrossRef]

30. Sootsman, J.R.; Kong, H.; Uher, C.; D’Angelo, J.J.; Wu, C.I.; Hogan, T.P.; Caillat, T.; Kanatzidis, M.G. Large enhancements in the thermoelectric power factor of bulk $\mathrm{PbTe}$ at high temperature by synergistic nanostructuring. Angew. Chem. Int. Ed. Engl. 2008, 47, 8618-8622. [CrossRef]

31. Pathan, H.M.; Lokhande, C.D. Deposition of metal chalcogenide thin films by successive ionic layer adsorption and reaction (SILAR) method. Bull. Mater. Sci. 2004, 27, 85-111. [CrossRef]

32. Komissarova, T.; Khokhlov, D.; Ryabova, L.; Dashevsky, Z.; Kasiyan, V. Impedance of photosensitive nanocrystalline PbTe (In) films. Phys. Rev. B. 2007, 75, 195326. [CrossRef]

33. Dong, P.; Wang, X.; Li, S.; Ni, Y. The role of $\mathrm{HNO}_{3}$ in the electrochemical deposition of dendritic PbTe microstructures. CrystEngComm 2018, 20, 1042-1049. [CrossRef]

34. Bae, S.; Kim, H.; Lee, H.S. Formation mechanism of PbTe dendritic nanostructures grown by electrodeposition. Mater. Chem. Phys. 2017, 187, 82-87. [CrossRef]

35. Wang, Y.Y.; Cai, K.F.; Yao, X. Facile synthesis of PbTe nanoparticles and thin films in alkaline aqueous solution at room temperature. J. Solid State Chem. 2009, 182, 3383-3386. [CrossRef]

36. Bhalekar, V.P.; Pathan, H.M. Strong Quantum Confined Lead Sulphide Quantum Dots using Ionic Reaction and Their properties. ES Mater. Manuf. 2019, 3, 52-56. [CrossRef]

37. Patil, R.S.; Pathan, H.M.; Gujar, T.P.; Lokhande, C.D. Characterization of chemically deposited nanocrystalline PbS thin films. J. Mater. Sci. 2006, 41, 5723-5725. [CrossRef] 
38. Kale, R.B.; Sartale, S.D.; Ganesan, V.; Lokhande, C.D.; Lin, Y.-F.; Lu, S.-Y. Room temperature chemical synthesis of lead selenide thin films with a preferred orientation. Appl. Surf. Sci. 2006, 253, 930-936. [CrossRef]

39. Pop, I.; Nascu, C.; Ionescu, V.; Indrea, E.; Bratu, I. Structural and optical properties of PbS thin films obtained by chemical deposition. Thin Solid Film 1997, 307, 240. [CrossRef]

40. Rempel, A.A.; Kozhevnikova, N.S.; Leenaers, A.J.G.; van den Berghe, S. Towards particle size regulation of chemically deposited lead sulfide (PbS). J. Cryst. Growth 2005, 280, 300-308. [CrossRef]

41. Ghamsari, M.S.; Araghi, M.K.; Farahani, S.J. The influence of hydrazine hydrate on the photoconductivity of PbS thin film. Mater. Sci. Eng. B 2006, 133, 113-116. [CrossRef]

42. Yang, Y.J.; Hu, S.S. The deposition of highly uniform and adhesive nanocrystalline PbS film from solution. Thin Solid Film 2008, 516, 6048-6051. [CrossRef]

43. Gorer, S.; Albu-Yaron, A.; Hodes, G. Chemical Solution Deposition of Lead Selenide Films: A Mechanistic and Structural Study. Chem. Mater. 1995, 7, 1243. [CrossRef]

44. Gorer, S.; Albu-Yaron, A.; Hodes, G. Quantum Size Effects in Chemically Deposited, Nanocrystalline Lead Selenide Films. J. Phys. Chem 1995, 99, 16442. [CrossRef]

45. Zhang, W.X.; Yang, Z.H.; Liu, J.W.; Qian, Y.T.; Yu, W.C.; Jia, Y.B.; Liu, X.M.; Zhou, G.; Zhu, J.S. A Simple Synthesis of Nanocrystalline Binary Metal Chalcogenides in Alkaline Aqueous Solution. Solid State Chem. 2001, 161, 184-189. [CrossRef]

46. Shi, X.-L.; Zou, J.; Chen, Z.-G. Advanced Thermoelectric Design: From Materials and Structures to Devices. Chem. Rev. 2020, 1-117. [CrossRef]

47. Jia, B.H.; Jiang, B.B.; He. J.Q. Recent advances of n-type low-cost PbSe-based thermoelectric materials. Mater. Today Adv. 2019, 4, 100029. [CrossRef]

48. Qin, Y.; Xiao, Y.; Wang, D.; Qin, B.; Huang, Z.; Zhao, L.-D. An approach of enhancing thermoelectric performance for p-type PbS: Decreasing electronic thermal conductivity. J. Alloys Compd. 2020, 820, 153453. [CrossRef]

49. Zhou, M.; Li, J.-F.; Kita, T. Nanostructured AgPbmSbTem+2 System Bulk Materials with Enhanced Thermoelectric Performance. J. Am. Chem. Soc. 2008, 130, 4527-4532. [CrossRef]

50. Wang, W.; Poudel, B.; Wang, D.; Ren, Z. Synthesis of PbTeNanoboxes Using a Solvothermal Technique. Adv. Mater. 2005, 17, 2110-2114. [CrossRef]

51. Zhang, G.; Lu, X.; Wang, W.; Li, X. Facile Synthesis of a Hierarchical PbTe Flower-like Nanostructure and Its Shape Evolution Process Guided by a Kinetically Controlled Regime. Chem. Mater. 2007, 19, 5207-5209. [CrossRef]

52. Murphy, J.E.; Beard, M.C.; Norman, A.G.; Ahrenkiel, S.P.; Johnson, J.C.; Yu, P.; Mićić, O.I.; Ellingson, R.J.; Nozik, A.J. PbTe Colloidal Nanocrystals: Synthesis, Characterization, and Multiple Exciton Generation. J. Am. Chem. Soc. 2006, 128, 3241-3247. [CrossRef] [PubMed]

53. Qiu, X.F.; Lou, Y.B.; Samia, A.C.S.; Devadoss, A.; Burgess, J.D.; Dayal, S.; Burda, C. PbTenanorods by sonoelectrochemistry. Angew. Chem. Int. Ed. 2005, 44, 5855-5857. [CrossRef]

54. Gite, A.B.; Palve, B.M.; Gaikwad, V.B.; Jain, G.H.; Pathan, H.M. Synthesis and characterization of electrodeposited lead telluride films on copper and stainless steel substrate. Mater. Res. Express 2019, 6. [CrossRef]

55. Martin-Gonzales, M.S.; Prieto, A.L.; Gronsky, R.; Sands, T.; Stacy, A.M. Insights into the electrodeposition of $\mathrm{Bi}_{2} \mathrm{Te}_{3}$. J. Electrochem. Soc. 2002, 149, C546-C554. [CrossRef]

56. Haram, S.K.; Quinn, B.M.; Bard, A.J. Electrochemistry of CdS Nanoparticles: A Correlation between Optical and Electrochemical Band Gaps. J. Am. Chem. Soc. 2001, 123, 8860-8861. [CrossRef]

57. Ganguly, M.; Rout, S.K.; Sinha, T.P.; Sharma, S.K.; Park, H.Y.; Ahn, C.W.; Kim, I.W. Characterization and Rietveld Refinement of A-site deficient Lanthanum doped Barium Titanate. J. Alloys Compd. 2013, 579, 473-484. [CrossRef]

58. Kadel, K.; Kumari, L.; Wang, X.; Li, W.; Huang, J.Y.; Provencio, P.P. Synthesis and structure of undoped and indium-doped thermoelectric lead telluride nanoparticles. Nanoscale Res. Lett. 2014, 9, 227. [CrossRef]

59. Williamson, G.B.; Smallman, R.C. Dislocation densities in some annealed and cold-worked metals from measurements on the Xray Debye-Scherrer spectrum. Philos. Mag. 1956, 1, 34-36. [CrossRef]

60. Peters, J.L.; De Wit, J.; Vanmaekelbergh, D. Sizing Curve, Absorption Coefficient, Surface Chemistry, and Aliphatic Chain Structure of PbTe Nanocrystals. Chem. Mater. 2019, 31, 1672-1680. [CrossRef] 
61. Pawar, S.B.; Shaikh, J.S.; Devan, R.S.; Ma, Y.R.; Haranath, D.; Bhosale, P.N.; Patil, P.S. Facile and low cost chemosynthesis of nanostructured PbS with tunable optical properties. Appl. Surf. Sci. 2011, 258, 1869-1875. [CrossRef]

62. Pan, Y.; Bai, H.; Pan, L.; Li, Y.; Tamargo, M.C.; Sohel, M.; Lombardi, J.R. Size controlled synthesis of monodisperse PbTe quantum dots: Using oleylamine as the capping ligand. J. Mater. Chem. 2012. [CrossRef]

63. Nithiyanantham, U.; Ozaydin, M.F.; Tazebay, A.S.; Kundu, S. Low-temperature formation of rectangular PbTe nanocrystals and their thermoelectric properties. New J. Chem. 2016, 40, 265-277. [CrossRef]

64. Tzounis, L.; Liebscher, M.; Fuge, R.; Leonhardt, A.; Mechtcherine, V. P- and n-type thermoelectric cement composites with CVD grown p- and n-doped carbon nanotubes: Demonstration of a structural thermoelectric generator. Energy Build. 2019. [CrossRef]

65. Crocker, A.J. The role of sodium in lead telluride. J. Phys. Chem. Solids 1967, 28, 1903-1912. [CrossRef]

66. Ito, M.; Seo, W.S.; Koumoto, K.; Mater, J. Thermo-electric properties of PbTe thin films prepared by gas evaporation method. J. Mater. Res. 1999, 14, 209. [CrossRef]

67. Zhu, P.W.; Chen, L.X.; Jia, X.; Ma, H.A.; Ren, G.Z.; Guo, W.L.; Zhang, W.; Zou, G.T. Thermoelectric properties of PbTe prepared at high pressure and high temperature. J. Phys. Condens. Matter 2002, 44, 11185-11188. [CrossRef]

(C) 2020 by the authors. Licensee MDPI, Basel, Switzerland. This article is an open access article distributed under the terms and conditions of the Creative Commons Attribution (CC BY) license (http://creativecommons.org/licenses/by/4.0/). 